\title{
Electron density measurements in shock tube using microwave interferometry
}

Cite as: Rev. Sci. Instrum. 90, 054706 (2019); https://doi.org/10.1063/1.5086854

Submitted: 24 December 2018 . Accepted: 24 April 2019 . Published Online: 16 May 2019

Nesrine Toujani (D), Awad Bin Saud Alquaity (D), and Aamir Farooq (D)

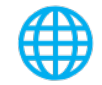

\section{ARTICLES YOU MAY BE INTERESTED IN}

Error correction associated with stray light for Faraday-effect polarimetry system on EAST Review of Scientific Instruments 90, 053501 (2019); https://doi.org/10.1063/1.5089699

A fully automated dual-tip scanning near-field optical microscope for localized optical excitation and detection in the visible and near-infrared

Review of Scientific Instruments 90, 053705 (2019); https://doi.org/10.1063/1.5084946

Potential clinical applications of terahertz radiation

Journal of Applied Physics 125, 190901 (2019); https://doi.org/10.1063/1.5080205

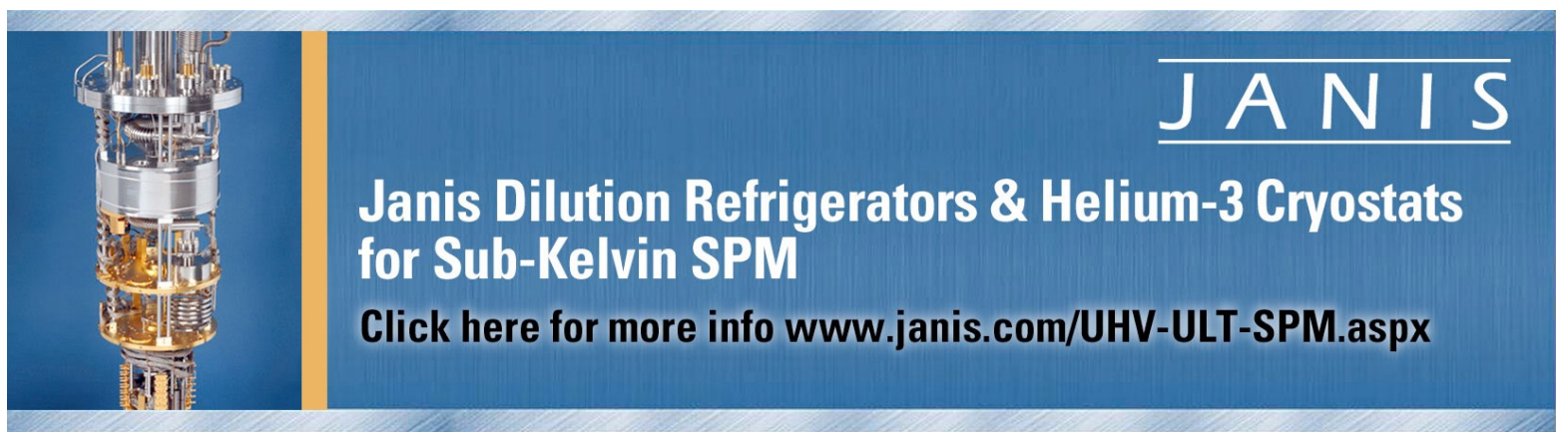




\title{
Electron density measurements in shock tube using microwave interferometry
}

\author{
Cite as: Rev. Sci. Instrum. 90, 054706 (2019); doi: 10.1063/1.5086854 \\ Submitted: 24 December 2018 • Accepted: 24 April 2019 • \\ Published Online: 16 May 2019
}

\begin{abstract}
Nesrine Toujani,' (D) Awad Bin Saud Alquaity, ${ }^{1,2}$ (D) and Aamir Farooq
AFFILIATIONS

${ }^{1}$ King Abdullah University of Science and Technology (KAUST), Clean Combustion Research Center, Physical Science and Engineering Division, Thuwal 23955, Saudi Arabia

${ }^{2}$ Rheinisch-Westfälische Technische Hochschule (RWTH) Aachen University, Institute for Combustion Technology, Templergraben 64, 52062 Aachen, Germany
\end{abstract}

a) Electronic mail: Aamir.farooq@kaust.edu.sa

\begin{abstract}
Microwave interferometry (MWI) is a nonintrusive diagnostic technique, capable of measuring small quantities of electrons present in a flame plasma. In this paper, a $94 \mathrm{GHz}$ microwave interferometer is characterized and validated to perform robust and reliable measurements of electron concentrations in thermal and nonthermal plasmas in a shock tube. The MWI system is validated first by measuring the refractive index of a dielectric material. Subsequently, the system is used for measuring electron densities during the thermal ionization of argon and krypton in shock tube experiments. The measured activation energies are in good agreement with both the measured values from previous studies and theoretical values. The MWI system is finally used for measuring electron density time-histories in fuel oxidation experiments in the shock tube. The electron density profile of methane combustion shows a peak at the ignition time which agrees with pressure measurements. Experimental electron histories are also in overall agreement with predictions of the methane ion chemistry model.
\end{abstract}

Published under license by AIP Publishing. https://doi.org/10.1063/1.5086854

\section{INTRODUCTION}

Electrical aspects of flames have recently gained considerable attention due to the possibility of manipulating the flame behavior by applying external electric fields. ${ }^{1-3}$ Flames and other combustion systems contain a small, yet non-negligible, quantity of electrons due to the phenomenon of chemi-ionization. This phenomenon results in the formation of ions or charged particles by chemical reactions. Determination of the charge carrier density is an important characterization in ionized media such as flames and plasmas.

Numerous techniques have been employed to detect electron densities in ionized media. The most widely used method is the Langmuir probe. To apply this technique, a probe electrode is inserted into the plasma. By recording the current-voltage plot, it is possible to determine the densities and energies of the charged particles. ${ }^{4}$ However, this kind of electrostatic probe is not very accurate and affects the measured density due to its invasive nature.

Optical emission spectroscopy is another laboratory diagnostic tool for plasma processes. This method determines electron density from the Stark broadening of the measured spectral lines. ${ }^{5}$ However, for relatively low electron densities in a shock tube, this technique is known to be unreliable because the electron number density is much lower compared to plasma applications.

Another largely used technique is Thomson scattering ${ }^{8}$ which is particularly valuable in the case of high-pressure plasma. It is based on the scattering of electromagnetic radiation by electrons. Scattering of laser light by solid surfaces and/or other particles present in the plasma can perturb this measurement.

Microwave interferometry (MWI) is another method that has been used extensively to measure electron densities in low-density plasmas. Compared to the previously mentioned techniques for electron density measurements, MWI is noninvasive, has higher accuracy, and allows easier interpretation of results. It determines the electron density in the plasma by measuring the phase shift and attenuation of the transmitted microwave signal. Cappelli et al. ${ }^{9}$ and Gilchrist et al. ${ }^{10}$ used microwave interferometry to measure the electron number density in a plasma plume. In addition, this technique has been applied to shock tube studies of thermal ionization of inert gases. In this context, several studies ${ }^{11-14}$ discussed 
TABLE I. Frequencies of MWI used in the literature.

\begin{tabular}{lc}
\hline \hline & MWI frequency $(\mathrm{GHz})$ \\
\hline Harwell and Jahn $^{11}$ & 24 \\
Kelly $^{12}$ & 24 \\
Schneider and Park $^{14}$ & 35 \\
Vlasov $^{18}$ & 37.5 \\
Cappelli et al. $^{9}$ & 90 \\
KAUST (this work) & 94 \\
\hline
\end{tabular}

experimental investigations of the rate of ionization of argon, krypton, and xenon behind shock waves.

MWI has also been used in other studies ${ }^{15-18}$ to study the chemical ionizations of hydrocarbon fuels such as methane and acetylene behind reflected shock waves. Experimental and kinetic simulation results were reported to be in close quantitative and qualitative agreement.

In this work, we have designed and applied a microwave interferometer for the investigation of chemi-ionization processes in combustion systems. A microwave interferometer was custombuilt for this purpose by Millitech ${ }^{19}$ to fit onto the shock tube at KAUST. ${ }^{20,21}$ The system generates microwaves at $94 \mathrm{GHz}$. Transmitting high frequency allows electron density measurements with higher spatial resolution compared to previous studies ${ }^{11,12,14,18}$ (see Table I). Spatial resolution is enhanced at shorter wavelengths, ${ }^{22,23}$ and this allows the MWI system to detect the spatial details of electron population.

The microwave interferometer outputs in-phase and quadraturephase signals. Plotting both signals as a function of one another, at controlled phase shift increments, results in a calibration Smith diagram. The phase shift can subsequently be used to calculate the electron density. The studies of Cappelli et al. ${ }^{9,24}$ report an elliptical shape of the Smith diagram instead of a circle. This distortion is explained by imbalances that are known to corrupt signal quality. The signal impurities have to be corrected for proper data interpretation. ${ }^{25,26}$ Imbalance compensations are thus carried out in this work, as described in Sec. III.

Here, we report three sets of measurements with the MWI system.

(1) In order to validate the MWI system, we carried out measurements of phase-shifts in a dielectric material with a known refractive index. Comparing the manufacturer data with the experimentally measured refractive index permits us to quantify the noise and verify the repeatability of the measurements.

(2) Next, thermal ionization studies of ideal gases, such as argon and krypton, are carried out in a shock tube. The method reliably produces ionized gases, and the results are compared against previously published data for ionization rates.

(3) Finally, electron concentration time-histories are measured during the oxidation of methane in the shock tube. The collected data demonstrate the usefulness of the developed MWI diagnostic for investigating chemi-ionization reactions of hydrocarbon fuels.

\section{THEORY}

\section{A. Microwave interferometry and electron concentration measurement}

The MWI is analogous to the Mach-Zehnder interferometer used in optics in the sense that they both need a reference and test branches. The interferometer splits a source beam into two paths: a test beam transmitted through the plasma and a reference beam propagating along a waveguide.

The free electrons present in the plasma alter the phase of the electromagnetic waves propagating in the measurement section. This results in a phase difference between the test path and the reference path of the interferometer. The electron density $n_{e}$ is then deduced from the measured phase shift $\Delta \phi$.

In the experiments described here, the effect of the magnetized field in the plasma is assumed negligible. The plasma frequency $\omega_{p}$ is much lower than the MWI's frequency $\omega$, which implies that lower plasma electron density $n_{e}$ compared to the critical density $n_{c}(3)$ is defined as the cutoff density above which waves cannot propagate. ${ }^{2}$ The relation between the phase shift $\Delta \phi$ and the electron density $n_{e}$ is described as ${ }^{28-31}$

$$
\Delta \phi=\frac{e^{2}}{2 \varepsilon_{0} m_{e} c \omega} \int n_{e}(x) d x \text { for } n_{e} \ll n_{c} .
$$

For a uniform plasma, the phase shift is linearly proportional to the average density along the path

$$
n_{e}=\frac{4 \pi \varepsilon_{0} m_{e} c^{2}}{\lambda e^{2} L} \Delta \phi
$$

where $m_{e}$ is the electron mass, $e$ is the electron charge, $c$ is the speed of light, $\lambda$ is the microwave wavelength, $\varepsilon_{0}$ is the free space permittivity, and $L$ is the path-length traversed by the microwave through the plasma.

\section{B. Thermal ionization}

The thermal ionization study of shock-heated noble gases refers to the study of the initial ionization process. It corresponds to the amount of energy required to remove the most loosely bound electron.

In the late 1960s, Harwell and Jahn, ${ }^{11}$ Kelly, ${ }^{12}$ and McLaren and Hobson ${ }^{13}$ used MWI to determine the ionization rates of argon, krypton, and xenon. In their work, they affirmed that the electron generation process proceeds in two steps, i.e., excitation and ionization according to the equations

$$
\begin{array}{rr}
A+A \rightarrow A+A^{*} & \text { excitation, } \\
A+A^{*} \rightarrow A+A^{+}+e & \text { ionization, }
\end{array}
$$

where $A^{*}$ is the excited state and $A^{+}$is the ion. ${ }^{12}$

Their experimental investigations determined the activation energies, which correspond to the first excitation potentials rather than the ionization potentials of the gases (see Table II). Quadratic dependence of the ionization rate on pressure was also demonstrated. 
TABLE II. Comparison of ionization and first excitation energies of $\mathrm{Ar}$ and $\mathrm{Kr}$ with literature data.

\begin{tabular}{lcc}
\hline \hline & Argon (eV) & Krypton (eV) \\
\hline Ionization energy First excitation energy $^{11,12,32}$ & 15.75 & 14 \\
Kelly $^{12}$ & $11.55-11.83$ & $9.91-10.62$ \\
Harwell and Jahn $^{11}$ & $11.2 \pm 0.9$ & $10.1 \pm 0.7$ \\
McLaren and Hobson $^{13}$ & $10.9 \pm 0.5$ & $10.4 \pm 0.5$ \\
\hline \hline
\end{tabular}

It is established that the slope of $\ln \left(d n_{e} / d t\right)$ vs $1 / k T$ is approximately equivalent to the activation energy of the ionization process

$$
\frac{d\left[\ln \frac{d n_{e}}{d t}\right]}{d(1 / k T)} \approx-E_{a} \quad \text { for } \quad k T \ll E_{a},
$$

where $k$ is the Boltzmann constant, $T$ is the temperature, $E_{a}$ is the activation energy of the studied gas, and $d n_{e} / d t$ is the initial slope of the electron density $n_{e}$ corresponding to the atom-atom generation rate at early times of the thermal ionization experiment.

It is worth noting that, after the determination of the initial slope, quadratic normalization corrections are applied. They compensate for small variation of neutral particle density caused by variations in the pressure.

\section{APPARATUS}

\section{A. Microwave interferometer}

The MWI used in this work is shown schematically in Fig. 1; it is custom-designed by Millitech, Inc. for application to the shock tube facility at KAUST. ${ }^{20,21}$ It is an extremely high frequency (EHF) system operating at $94 \mathrm{GHz}$ (wavelength $\lambda=3.2 \mathrm{~mm}$ ). At this frequency, the system's critical density is

$$
n_{c}=\frac{\omega^{2} \varepsilon_{0} m_{e}}{e^{2}}=1.1 \times 10^{20} \mathrm{~m}^{-3} .
$$

The sensitivity of the MWI is defined as the minimum detectable density change. It depends on both the MWI's signal and the DAQ system used for the data acquisition. It corresponds to a change of one level of the DAQ. ${ }^{33}$ In the present work, the system is set at 14 bits, and every level corresponds to $10 \mu \mathrm{V}$. Let $k$ be the scale factor relating the change in signal amplitude to the change in phase. By determining $k$, one can estimate the change in phase corresponding

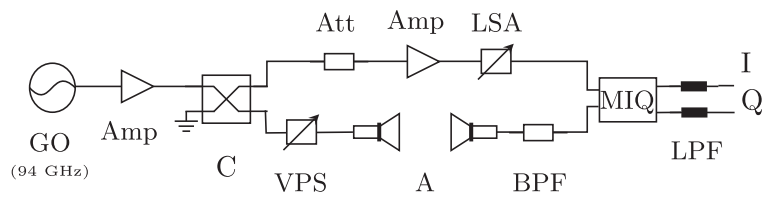

FIG. 1. Schematic diagram of the microwave interferometer. GO: Gunn Oscillator; Amp: amplifier; C: coupler; Att: attenuator; LSA: Level Set Attenuator; VPS: Variable Phase Shifter; A: horn antenna; BPF: Bandpass Filter; MIQ: Mixer I/Q; and LPF: Low Pass Filter. to $10 \mu \mathrm{V}$. As a result, the minimum change in phase detected by the MWI is $0.17^{\circ}$, and the corresponding density using Eq. (1) is 1.56 $\times 10^{15} \mathrm{~m}^{-3}$.

The microwaves are generated by a Gunn diode oscillator. They are amplified and split into a reference and test wave. The reference wave goes through a reference leg. The test wave travels through the desired plasma from a transmitting to receiving horn-antenna. Both antennas are physically and electromagnetically symmetrical and are separated by $157 \mathrm{~mm}$. Test and reference waves are then combined in the system's I/Q mixer to measure the relative phase shift between the two waves. The output signals are the in-phase I and the quadrature phase $\mathrm{Q}$. The resulting signals then go through low pass filters in order to attenuate the noise outside the desired bandwidth.

The MWI is equipped with a level set attenuator that adjusts the waves' attenuation up to $25 \mathrm{~dB}$ and a variable phase shifter that allows up to $180^{\circ}$ phase shift. These two components are essential to calibrate the MWI prior to each experiment.

\section{Calibration of the MWI}

By varying the phase shifter in increments, the recorded I and Q signals define two sinusoidal oscillations with a $90^{\circ}$ offset from one another.

Plotting the two waves in the Cartesian coordinate as a function of one another results in a calibration Smith circle representative of $360^{\circ}$ phase shift.

However, due to hardware imperfections, the difference between the output of the I/Q mixer and signal paths causes amplitude and phase imbalance. As a result, the calibration circle is distorted into an ellipse, as shown in Fig. 2, and could lead to incorrect phase shift determination.

In this context, Singh et al. ${ }^{25}$ proposed an efficient and accurate data-based quadrature imbalance correction method without any hardware modification. The amplitude $A_{e}$ and phase imbalance $\Delta \phi_{e}$ coefficients are determined from the best-fit ellipse and then used to perform an orthonormalization transformation known as Gram-Schmidt procedure.

After imbalance correction and fitting for the DC offset, the size of the calibration circles is indicative of the attenuation level, as illustrated in Fig. 3. Note also that the variable attenuator introduces an additional phase shift that has to be canceled when carrying out the experiments. For the measurements reported here, the variable attenuator is set at zero attenuation.

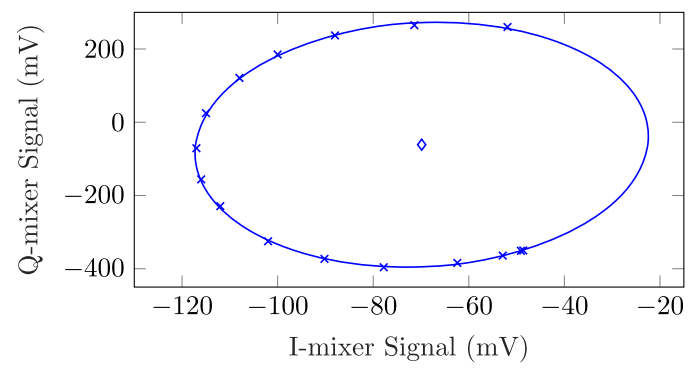

FIG. 2. Smith diagram of MWl's response to calibration. 


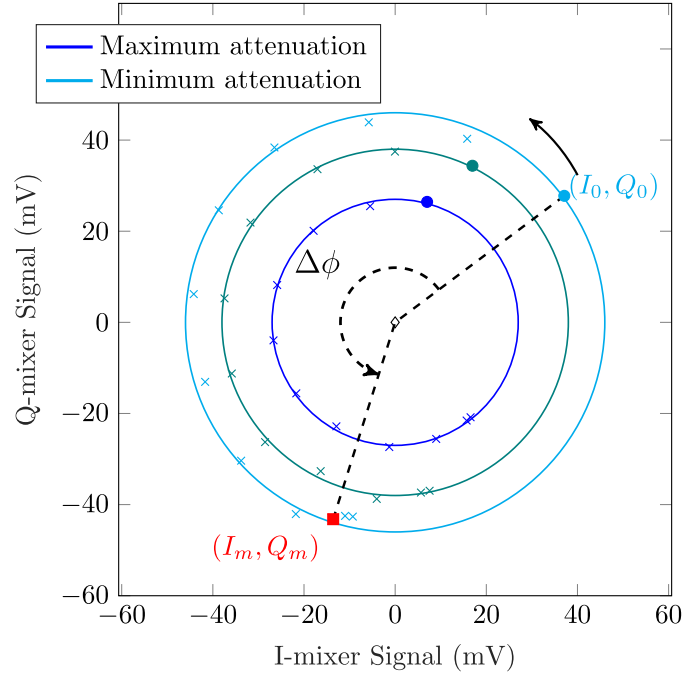

FIG. 3. Smith diagram of MWI's response after imbalance compensations

\section{Beamwidth of the MWI}

The beamwidth of the MWI is characterized in both horizontal and vertical planes. To this end, a dielectric material is translated, in both horizontal and vertical planes, along the distance between the horn antennas. When the MWI starts to respond to the presence of the material in the transmission beam, data points are recorded and considered as the edge of the transmission pattern.

Measurements indicate a diverging MWI transmission beam with side lobes in the near field of both horn antennas as illustrated in Fig. 4.

The measured beam width reaches approximately $67 \mathrm{~mm}$ in the horizontal plane (XY plane) and $40 \mathrm{~mm}$ in the vertical plane (plane $\mathrm{YZ}$ ) of the MWI transmission pattern.

\section{Validation of the MWI}

To validate the MWI, a material with known refractive index is used and the measured refractive index is compared with the manufacturer reported value. This step is important to quantify the noise in amplitude and phase and to verify the repeatability of the

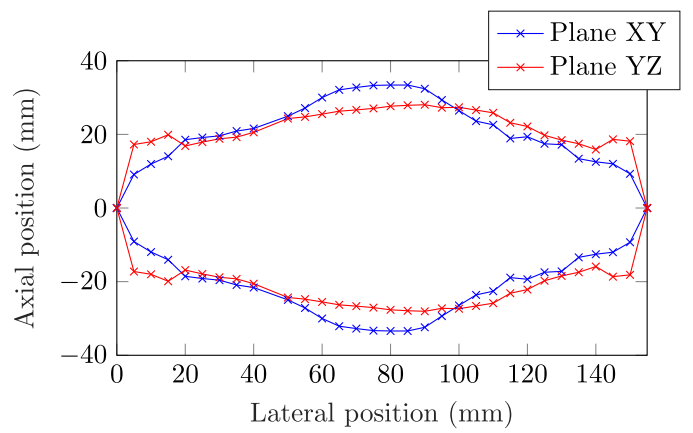

FIG. 4. Beamwidth of the MWI in the vertical and horizontal planes.
TABLE III. Comparison of experimental and referenced Rexolite refractive index for 6 measurements.

\begin{tabular}{lcc}
\hline \hline & Phase shift $\Delta \phi(\mathrm{deg})$ & Refractive index $\eta$ \\
\hline Manufacturer data & 856.15 & 1.59 \\
Experimental mean & 935.32 & 1.65 \\
Standard deviation $\sigma$ & 4.56 & 0.02 \\
\hline \hline
\end{tabular}

measurements. To that end, a $13 \mathrm{~mm}$-thick Rexolite ${ }^{34}$ dielectric material was used for the validation measurements.

The dielectric constant $\varepsilon_{r}$ of the material and its refractive index $\eta$ depend on the measured phase shift and material thickness.

The phase shift $\Delta \Phi$ introduced by the dielectric material is expressed as

$$
\Delta \Phi=\Delta \phi+n * 2 \pi
$$

where $n$ is a positive integer (to be determined) and $\Delta \phi \in[0 ; 2 \pi]$ is the phase-angle of the MWI. Using the calibration circle shown in Fig. 3, $\Delta \phi$ corresponds to the phase difference between the data point $\left(I_{0}, Q_{0}\right)$, measured at $0^{\circ}$ phase and without any sample in the transmission beam, and the data point $\left(I_{m}, Q_{m}\right)$ measured in the presence of the material sample.

For materials with known refractive index, Trabelsi et al. ${ }^{35}$ established the following relation to determine either the thickness of the material $d$ or the integer $n$ :

$$
\left(n-\frac{1}{2}\right) \frac{\lambda}{\sqrt{\varepsilon_{r}}-1} \leq d \leq\left(n+\frac{1}{2}\right) \frac{\lambda}{\sqrt{\varepsilon_{r}}-1} .
$$

This method solves the problem referred to as the "phase ambiguity." It is encountered when the thickness of the material is greater than the MWI wavelength.

Using the Rexolite material, measurements gave repeatable results (Table III), with errors less than $10 \%$ compared to the manufacturer value.

\section{B. Shock tube}

Thermal ionization and methane oxidation experiments are performed in the KAUST low pressure shock tube facility. ${ }^{20}$ The shock tube is constructed from stainless steel with an internal diameter of $142 \mathrm{~mm}$. It consists of a $9 \mathrm{~m}$ long driven section filled with the test gas and a modular driver section filled with high-pressure helium. The maximum length of the driver section is $9 \mathrm{~m}$. The internal surface of the shock tube is honed and electropolished to minimize the wall impurities.

All experiments are performed behind reflected shock waves. For each run, the driver and the driven sections of the shock tube are separated by a polycarbonate diaphragm placed in front of a square cutter blade to burst the diaphragms in a controlled manner. The thickness of the diaphragm and the position of the cutting blade are adjusted prior to each experiment according to the target temperature and pressure behind the reflected shock wave.

The MWI horn antennas are placed $2 \mathrm{~cm}$ away from the driven section endwall. The arrival of the reflected shock wave at this location marks the beginning of the test time.

The conventional sidewall windows of the shock tube are replaced with ports made of PVC to accommodate the MWI horn 


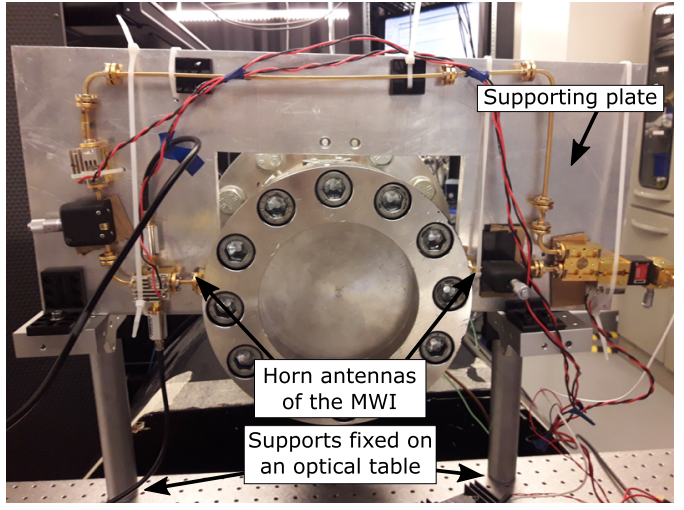

FIG. 5. Setup of the MWI on the shock tube.

antennas. Mounting the MWI directly on the shock tube generated continuous noise in the output signal. The sources of this noise are small vibrations in the shock tube that happen during pumping down, switching valves, and arrival of shock waves. To avoid this issue, the MWI supporting plate is mounted on a separate optical table. It eliminates contact between the MWI and the shock tube, while keeping the horn antennas floating in the shock tube ports, as shown in Fig. 5.

Incident shock velocities are measured using five piezoelectric PCB pressure transducers, located axially along the last $1.5 \mathrm{~m}$ of the shock tube. Shock speed is linearly extrapolated to the endwall in order to determine the reflected shock temperatures and pressures using standard normal shock relations. The overall temperature uncertainty is estimated to be $\pm 0.9 \%$. It is mainly dominated by uncertainty of the measured shock speed.

The experimental test mixtures are manometrically prepared in a gas-mixing facility of a magnetically stirred mixing tank and a 14port gas-mixing manifold using three MKS pressure gauges located at different ports.

\section{RESULTS AND DISCUSSION}

\section{A. Thermal ionization of argon and krypton}

Thermal ionization of argon and krypton is studied using the MWI in the shock tube to measure the electron density in the early stages of the ionization process. The results are compared with previously published studies. ${ }^{11-14}$

The objective is to determine the activation energy of monoatomic ideal gases such as argon (Grade 6) and krypton (Grade 5), supplied by Air Liquide. During the experiment, the MWI detects the production of electrons caused by the thermal ionization process. An increase in plasma density is observed as the phase shift in the signals plotted on an I/Q plot (Fig. 6). The increase in plasma density leads to the formation of circles with decreasing radius representing phase shift and attenuation due to the increase in electron density. A typical corrected signal evolution recorded in this experiment is shown in Fig. 6.

Some deviations from the ideal circle, in addition to distortion loops, are commonly observed on the I/Q signal during thermal ionization. This is due to the reflection and scattering of the

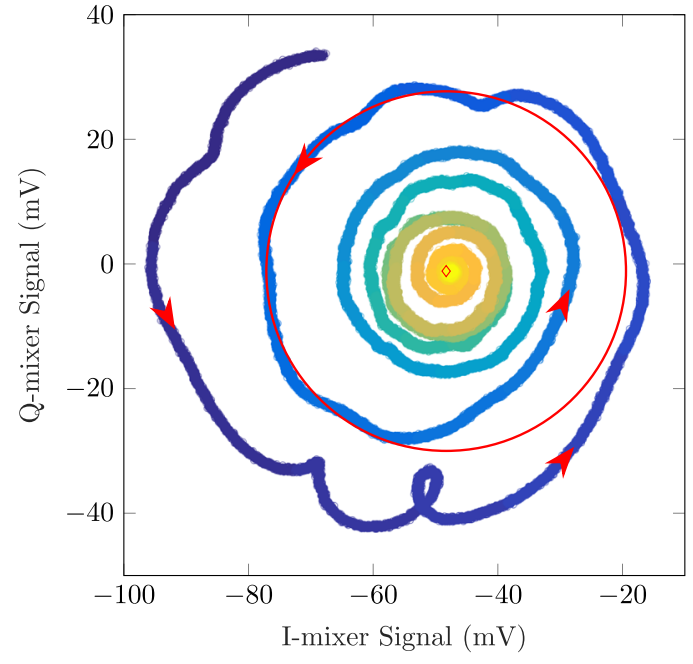

FIG. 6. Evolution of the microwave signal during the shock experiment with krypton at $6590 \mathrm{~K}$ and 0.1 bar. Red curve is the calibration circle.

microwave signal at boundaries/surfaces inside the system. The reflections generate a second signal with smaller intensity received by the interferometer. ${ }^{3}$

The derived phase shift is converted to electron density using Eq. (1). In Fig. 7, plotting the evolution of electron concentration during the early times of the thermal ionization process shows a transitory plateau that has been observed in previous studies. ${ }^{12,14}$ The initial slope $d n_{e} / d t$ is obtained from the linear part of the plot. Beyond this initial portion, the evolution of the trace is no longer linear due to increasing effects of shock attenuations by electron generation.

For each gas, the values of the measured initial slopes of thermal ionization experiments are quadratically normalized to the pressure behind reflected shock wave P5. Then, activation energy $E_{a}$ is derived from the slope of the best fitting line of the Arrhenius plot of $d n_{e} / d t$ vs $1 /(k T)$, as shown in Figs. 8 and 9. The obtained slopes are comparable to those reported in earlier studies.

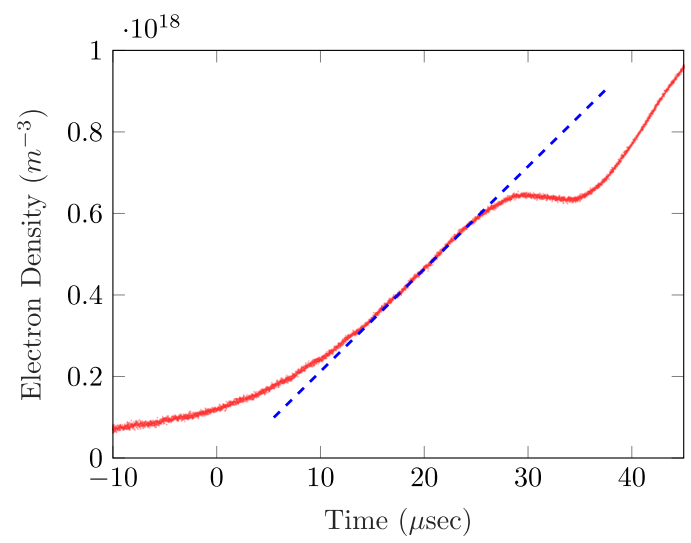

FIG. 7. Variation in electron density of shock heated argon at $7083 \mathrm{~K}$ and 0.09 bar Blue dashed line is the initial slope $d n_{e} / d t$. 


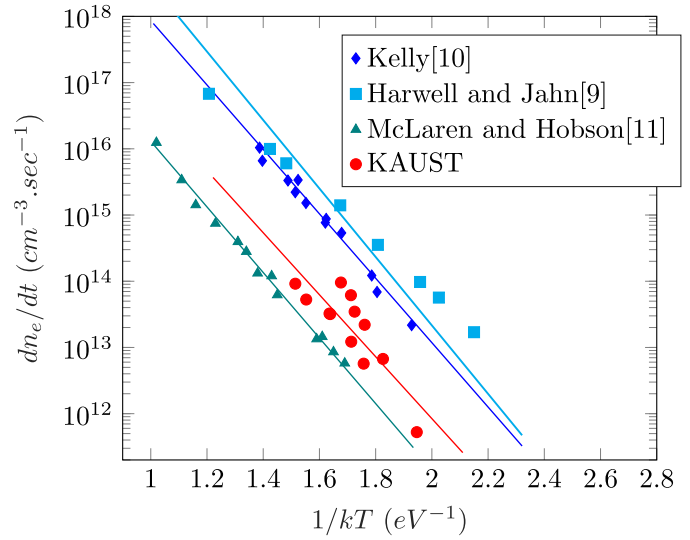

FIG. 8. Arrhenius plot of ionization of argon, quadratically normalized to P5 $=0.1$ bar and compared to literature data.

Some scatter in the results is seen at high temperatures due to impurities, as reported in previous studies. ${ }^{11}$ In this work, it is observed that impurity reaches levels higher than $3 \%$ for experiments conducted at high temperatures $(>8000 \mathrm{~K})$. This is due to the small value of initial pressure needed in the test section to obtain high temperatures behind reflected shock waves coupled with almost constant leak rates which increases the impurities at low initial pressures ( $<3$ mbar). As a result, obtained electron densities are quite scattered.

In addition, we consider the time required to reach the transition to electron-atom processes. It corresponds to the duration between the beginning of the experiment (pressure rise at the arrival of reflected shock) and the end of the transitory plateau corresponding to the initial slope of the electron density profile. Observations showed that the transition to electron-atom process becomes remarkably fast at high temperatures. Consequently, high temperature data $(>8000 \mathrm{~K})$ for both argon and krypton thermal ionization are disregarded.

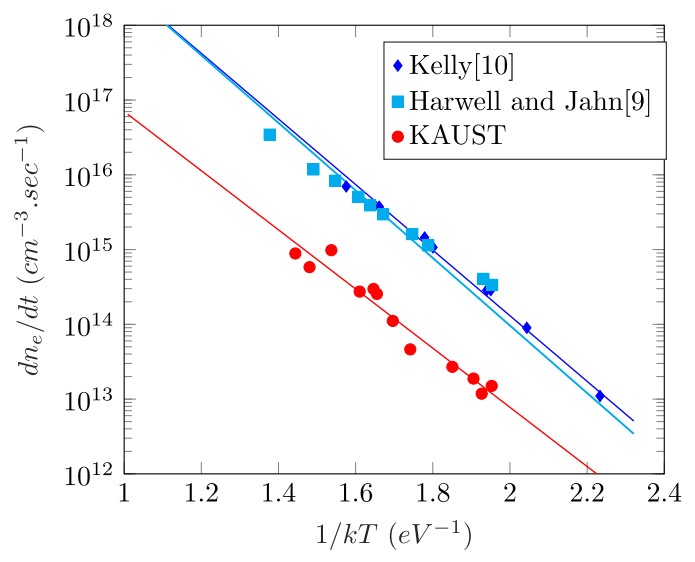

FIG. 9. Arrhenius plot of ionization of krypton, quadratically normalized to P5 $=0.99$ bar and compared to literature data.
TABLE IV: Experimental results of activation energies compared to the theoretical first excitation energies of $\mathrm{Ar}$ and $\mathrm{Kr}^{11,12,32}$

\begin{tabular}{lrr}
\hline \hline & \multicolumn{1}{c}{ KAUST } & \multicolumn{1}{c}{ Theory } \\
\hline Argon $E_{a}(\mathrm{eV})$ & $10.77 \pm 2.12$ & $11.55-11.83$ \\
Krypton $E_{a}(\mathrm{eV})$ & $9.11 \pm 0.65$ & $9.91-10.62$ \\
\hline \hline
\end{tabular}

The obtained results, shown in Table IV, are in fact coincident with the first excitation energies rather than the ionization energies. The MWI experimental results are comparable to literature values (see Table II). The difference can be explained by uncertainties resulting from the purity of the gases/shock tube, microwave reflections from the shock tube inner surface, determination of initial slope, and temperature calculation.

Overall, given the agreement with previous studies, the MWI designed in this work is confirmed to be a reliable diagnostic tool for electron detection. Thermal ionization of the noble gases represents a successful validation of the MWI to study the combustion kinetics in the shock tube.

\section{B. Combustion of hydrocarbon fuels in shock tube}

A major motivation of the present work is the characterization of combustion reactions with microwave interferometry. The chemi-ionization reaction $\mathrm{CH}+\mathrm{O} \rightarrow \mathrm{CHO}^{+}+\mathrm{e}^{-}$is believed to be the most prominent source of electrons in hydrocarbon combustion. ${ }^{15,37}$ Therefore, to demonstrate the potential of the aforementioned MWI diagnostic, electron concentrations are measured in a methane/oxygen mixture diluted in argon. The composition of the mixture is $2 \% \mathrm{CH}_{4}, 4 \% \mathrm{O}_{2}$, and $94 \%$ Ar.

As illustrated in Fig. 10, typical combustion experiments in the shock tube show the arrival of the incident shock wave at the observation location ( $2 \mathrm{~cm}$ from the endwall), marked by a rise in pressure. Shortly afterward, the shock wave reflects off the endwall of the driven section, and a second rise in pressure is observed when the reflected wave passes the measurement location. At this point,

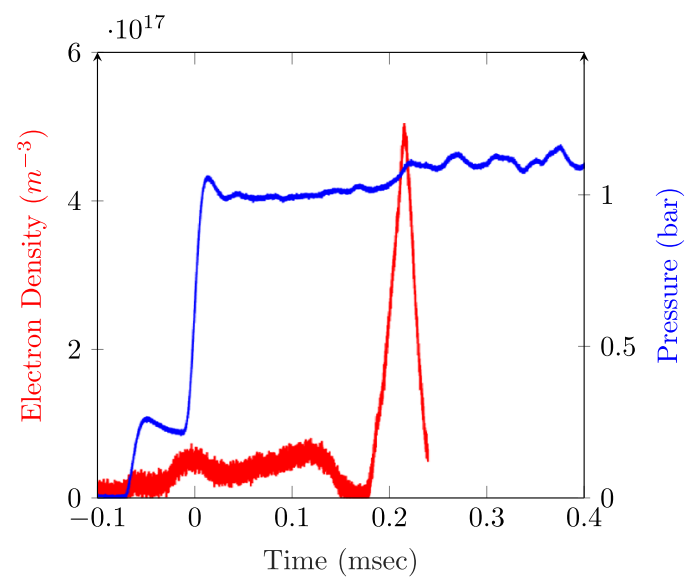

FIG. 10. Electron density during the stoichiometric combustion of methane at $1850 \mathrm{~K}$ and 0.98 bar. 
the air-fuel mixture is brought to the desired high-temperature and high-pressure conditions behind the reflected shock wave. After some delay, defined as the ignition delay time, a peak in electron density is observed at ignition. For experiments with high fuel concentration, a distinct pressure rise is seen at ignition and is used as an indicator of ignition timing. ${ }^{38}$ However, in dilute conditions or for low-reactivity fuels, pressure rise may not be very clear. In such situations, alternative indicators of ignition, such as chemiluminescence or electron density, could be used more effectively. In all experiments reported here, the time delay for peak electron concentrations coincides with the rise in pressure and can potentially be used as an alternate marker for measuring ignition delay times. This synchronization confirms the accuracy of our experimental measurements.

In most of the experiments, a small rise in electron density is observed before the peak appears. Despite the precautions taken, some noise appears in the MWI signal due to shock tube vibrations that accompany the arrival of a shock wave. However, such vibrations only occur for a short period of time and cease before the ignition of the fuel. Hence, it does not affect the measured electron density peak.

The experimental measurements of the electron density are compared with zero-dimensional simulations in Chemkin-Pro software. The simulations are based on the chemical kinetic model used by Alquaity et al. ${ }^{39}$ for methane oxidation. This model combines neutral molecule chemistry from AramcoMech ${ }^{40}$ and ion chemistry from Prager et al. ${ }^{41}$ The experimental electron densities are shown in Fig. 11 with their corresponding simulated predictions. Note that the peak electron concentration depends on temperature. The combustion process accelerates as temperature increases due to the Arrhenius nature of the reaction.

Simulated ignition delay times are a bit longer than the measured ones, except for the lowest temperature case. These differences

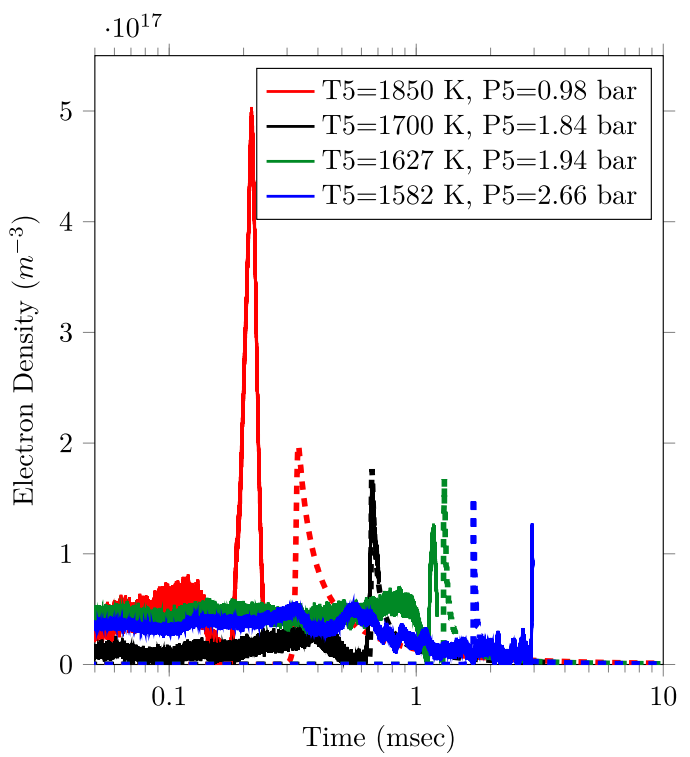

FIG. 11. Experimental electron densities (solid lines) compared to their simulated equivalent (dashed lines) for methane combustion reactions, normalized to P5 $=1.6$ bar. are largely due to the uncertainties in the gas phase chemistry mechanism used to model methane ignition. The simulations predict significantly lower peak electron concentration at $1850 \mathrm{~K}$. However, the predicted and measured peaks are in relatively good agreement in other cases. The discrepancy that exists between the measured and simulated electron concentrations is mainly due to inadequacies in the ion reaction mechanism, as pointed out by Alquaity et al. ${ }^{39}$

\section{SUMMARY}

Microwave interferometry is used for electron density measurements in ionized media such as flames and plasmas. The advantage of this method compared to the other techniques is that it is nonintrusive and measures electron density with higher spatial resolution.

In this paper, a microwave interferometer was designed to make electron concentration measurements in a shock tube. Imbalances of phase and amplitude due to hardware imperfections were corrected for better interpretation of results. Thereafter, validation was made with a dielectric material where the refractive index is measured experimentally and compared to the manufacturer data.

Thermal ionizations of argon and krypton were studied, and the experimental results were compared against literature data. Within the limit of accuracy, the measured activation energies of the inert gases were in agreement with the previous studies, despite the effect of impurities at high temperatures.

The driving purpose of this work was the characterization of hydrocarbon oxidation reactions with microwave interferometry. The steep rise in electron concentration at the time of ignition agreed with pressure measurements. The MWI diagnostic could be used as an alternative marker of ignition, particularly in dilute experiments where pressure rise is quite small. The measured electron concentrations are in overall good agreement with the predictions of a methane ion chemistry model. Future work will investigate the effect of fuel molecular structure and $\mathrm{C} / \mathrm{H}$ ratio on the peak electron densities.

\section{ACKNOWLEDGMENTS}

The work reported in this paper was funded by the Office of Sponsored Research (OSR) at the King Abdullah University of Science and Technology.

\section{REFERENCES}

${ }^{1}$ A. Feng and Z. Munir, "The effect of an electric field on self-sustaining combustion synthesis: Part I. Modeling studies,” Metall. Mater. Trans. B 26, 581-586 (1995).

${ }^{2}$ K. E. Shuler and J. Weber, "A microwave investigation of the ionization of hydrogen-oxygen and acetylene-oxygen flames," J. Chem. Phys. 22, 491-502 (1954).

${ }^{3}$ H. F. Calcote, "Ion and electron profiles in flames," Symp. (Int.) Combust. 9, 622-637 (1963)

${ }^{4}$ T. I. Cox, V. G. I. Deshmukh, D. A. O. Hope, A. J. Hydes, N. S. J. Braithwaite, and N. M. P. Benjamin, "The use of Langmuir probes and optical emission spectroscopy to measure electron energy distribution functions in RF-generated argon plasmas," J. Appl. Phys. 20, 820-831 (1987).

${ }^{5}$ K. A. Aadim, A. A.-K. Hussain, N. K. Abdalameer, H. A. Tawfeeq, and H. H. Murbat, "Electron temperature and density measurement of plasma jet in atmospheric pressure," Int. J. Novel Res. Phys. Chem. Math. 2, 28-32 (2015). 
${ }^{6}$ G. G. Gifford, "Applications of optical emission spectroscopy in plasma manufacturing systems," Proc. SPIE 1392, 454-465 (1990).

${ }^{7}$ D. M. Devia, L. V. Rodriguez-Restrepo, and E. Restrepo-Parra, "Methods employed in optical emission spectroscopy analysis: A review," Ing. Cienc. 11, 239-267 (2015).

${ }^{8}$ E. Carbone and S. Nijdam, "Thomson scattering on non-equilibrium low density plasmas: Principles, practices and challenges," Plasma Phys. Controlled Fusion 57, 014026 (2015).

${ }^{9}$ M. A. Cappelli, N. Gascon, and W. A. Hargus, Jr., "Millimetre wave plasma interferometry in the new field of a hall plasma accelerator," J. Phys. D: Appl. Phys. 39, $4582-4588$ (2006).

${ }^{10}$ B. E. Gilchrist, S. G. Ohler, and A. D. Gallimore, "Flexible microwave system to measure the electron number density and quantify the communications impact of electric thruster plasma plumes," Rev. Sci. Instrum. 68, 1189-1194 (1997).

${ }^{11}$ K. E. Harwell and R. G. Jahn, "Initial ionization rates in shock-heated argon, krypton and xenon," Phys. Fluids 7, 214-222 (1964).

${ }^{12}$ A. J. Kelly, "Atom-atom ionization cross sections of the noble gases-argon, krypton, and xenon," J. Chem. Phys. 45, 1723-1732 (1966).

${ }^{13}$ T. I. McLaren and R. M. Hobson, "Initial ionization rates and collision cross section in shock-heated argon," Phys. Fluids 11, 2162-2171 (1968).

${ }^{14}$ K.-P. Schneider and C. Park, "Shock tube study of ionization rates of $\mathrm{NaCl}-$ contaminated argon," Phys. Fluids 18, 969-981 (1975).

${ }^{15} \mathrm{Y}$. K. Karasevich, "Kinetics of chemical ionization in shock waves: Ionization kinetics in hydrocarbon oxidation," Kinet. Catal. 49, 610-615 (2008).

${ }^{16} \mathrm{Y}$. K. Karasevich, "Kinetics of chemical ionization in shock waves: Kinetic model of ionization in methane oxidation," Kinet. Catal. 50, 73-81 (2009).

${ }^{17} \mathrm{Y}$. K. Karasevich, "Kinetics of chemical ionization in shock waves: IV. Kinetic model of ionization in acetylene oxidation," Kinet. Catal. 50, 617-626 (2009).

${ }^{18}$ P. A. Vlasov, I. V. Zhiltsova, V. N. Smirnov, A. M. Tereza, G. L. Agafonov, and D. I. Mikhailov, "Chemical ionization of $\mathrm{n}$-hexane, acetylene, and methane behind reflected shock waves," Combust. Sci. Technol. 190, 57-81 (2018).

${ }^{19}$ See http://www. millitech.com for Millitech, Inc.; accessed December 2018.

${ }^{20}$ A. Alquaity, E. Es-sebbar, and A. Farooq, "Sensitive and ultra-fast species detection using pulsed cavity ringdown spectroscopy," Opt. Express 23, 7217 (2015).

${ }^{21}$ A. B. S. Alquaity, "Laser and mass spectrometric measurements of combustion species," Ph.D. thesis, King Abdullah University of Science and Technology, Thuwal, Kingdom of Saudi Arabia, 2016.

${ }^{22} \mathrm{~S}$. W. Janson, "Microwave diagnostics for ion engines," in 23rd International Electric Propulsion Conference Seattle, WA, IEPC-93-237 (AIAA, 1993), pp. 21852189.

${ }^{23}$ F. E. Coffield, S. R. Thomas, D. Lang, and R. D. Stever, "Microwave interferometer using 94-GHz solid-state sources," in 10th IEEE Symposium on Fusion Engineering (IEEE, 1983).

${ }^{24}$ G. D. Reed, W. A. Hargus, Jr., and M. A. Cappelli, "Microwave interferometry $(90 \mathrm{GHz})$ for hall thruster plume density characterization," in
AIAA/ASME/SAE/ASEE Joint Propulsion Conference, Tuscon, AZ (AIAA, 2005).

${ }^{25}$ A. Singh, X. Gao, E. Yavari, M. Zakrzewski, X. H. Cao, V. M. Lubecke, and O. Boric-Lubecke, "Data-based quadrature imbalance compensation for a CW Doppler radar system," IEEE Trans. Microwave Theory Tech. 61, 1718-1724 (2013).

${ }^{26}$ B.-K. Park, S. Yamada, and V. Lubecke, "Measurement method for imbalance factors in direct-conversion quadrature radar system," IEEE Microwave Wireless Compon. Lett. 17, 403-405 (2007).

${ }^{27}$ F. F. Chen, Plasma Physics and Controlled Fusion, Vol. 1: Plasma Physics (Plenum Press, New York and London, 1984).

${ }^{28} \mathrm{M}$. Ghorannevisse and M. Avakian, "Interferometry measurement of line average electron density in Alvand IIC tokamak," Int. J. Infrared Millimeter Waves 14, 17-22 (1993).

${ }^{29}$ M. Mirchner and J. C. H. Kruger, Partially Ionized Gases (Wiley, New York, 1973).

${ }^{30} \mathrm{~K}$. Dittmann, C. Kullig, and J. Meichsner, "160 GHz Gaussian beam microwave interferometry in low-density RF plasmas," Plasma Sources Sci. Technol. 21, 024001 (2012).

${ }^{31}$ O. Tudisco, A. L. Fabris, C. Falcetta, L. Accatino, R. D. Angelis, M. Manente, F. Ferri, M. Florean, C. Neri, C. Mazzota, D. Pavarin, F. Pollastrone, G. Rocchi, A. Selmo, L. Tasinato, F. Trezzolani, and A. A. Tuccillo, "A microwave interferometer for small and tenuous plasma density measurements," Rev. Sci. Instrum. 84, 033505 (2013).

${ }^{32}$ G. A. Cook, Argon, Helium, and the Rare Gases (Interscience, New York, 1961).

${ }^{33}$ J. Elizondo, D. Korneev, I. Nascimento, and W. de Sa, "TCABR interferometer," Braz. J. Phys. 32, 123-130 (2002).

${ }^{34}$ See http://www.rexolite.com for Rexolite; accessed December 2018.

${ }^{35}$ S. Trabelsi, A. W. Kraszewski, and S. O. Nelson, "Phase-shift ambiguity in microwave dielectric properties measurements," IEEE Trans. Instrum. Meas. 49, 56-60 (2000)

${ }^{36}$ G. Neumann, U. Banziger, M. Kammeyer, and M. Lange, "Plasma-density measurements by microwave interferometry and Langmuir probes in an RF discharge," Rev. Sci. Instrum. 64, 19-25 (1993).

${ }^{37}$ D. C. Murphy, "The measurement and application of electric effects in combustion," Ph.D. thesis, University of California, Berkeley, 2015.

${ }^{38}$ D. F. Davidson and R. K. Hanson, "Interpreting shock tube ignition data," in WSSCI Fall 2003 Meeting, 2003.

${ }^{39}$ A. B. Alquaity, C. Bingjie, J. Han, H. Selim, M. Belhi, Y. Karakaya, T. Kasper, S. M. Sarathy, F. Bisetti, and A. Farooq, "New insights into methane-oxygen ion chemistry," Proc. Combust. Inst. 36, 1213-1221 (2017).

${ }^{40}$ W. K. Metcalfe, S. M. Burke, S. S. Ahmed, and H. J. Curran, "A hierarchical and comparative kinetic modeling study of $\mathrm{C} 1-\mathrm{C} 2$ hydrocarbon and oxygenated fuels," Int. J. Chem. Kinet. 45, 638-675 (2013).

${ }^{41} \mathrm{~J}$. Prager, U. Riedel, and J. Warnatz, "Modeling ion chemistry and charged species diffusion in lean methane-oxygen flames," Proc. Combust. Inst. 31, 11291137 (2007). 